\title{
Une industrie au service du pain : les carrières de meules de Cinq-Mars-la-Pile (Indre-et-Loire), au $\mathrm{XVII}^{\mathrm{e}}$ siècle
}

An industry devoted to bread: the millstone quarries of Cinq-Mars-la-Pile (Indreet-Loire) in the seventeenth-century

\section{Alain Belmont}

\section{(2) OpenEdition}

\section{Journals}

Édition électronique

URL : http://journals.openedition.org/abpo/3141

DOI : 10.4000/abpo.3141

ISBN : 978-2-7535-4882-4

ISSN : 2108-6443

Éditeur

Presses universitaires de Rennes

Édition imprimée

Date de publication : 15 décembre 2015

Pagination : 47-66

ISBN : 978-2-7535-4880-0

ISSN : 0399-0826

\section{Référence électronique}

Alain Belmont, "Une industrie au service du pain : les carrières de meules de Cinq-Mars-la-Pile (Indreet-Loire), au XVII e siècle », Annales de Bretagne et des Pays de l'Ouest [En ligne], 122-4 | 2015, mis en ligne le 15 décembre 2017, consulté le 21 avril 2019. URL : http://journals.openedition.org/abpo/3141 ; DOI : 10.4000/abpo.3141 


\title{
Une industrie au service du pain : les carrières de meules de Cinq-Mars-la- Pile (Indre-et-Loire), au XVII ${ }^{\mathrm{e}}$ siècle
}

\author{
Alain BELMONT \\ Professeur d'histoire moderne, université Grenoble-Alpes, LARHRA UMR CNRS 5190
}

Situé au bord de la Loire et à 17 kilomètres en aval de Tours, le village de Cinq-Mars-la-Pile (Indre-et-Loire) a été rendu célèbre par un de ses seigneurs, le marquis Henri de Ruzé d'Effiat (1620-1642), exécuté sur une place de Lyon après avoir comploté contre Richelieu. Plus accessoirement, les archéologues connaissent Cinq-Mars pour sa "pile ", une haute tour de briques qui fut bâtie à l'époque romaine pour un usage funéraire. Mais ce que l'on sait moins, au point que même les auteurs de rapports statistiques d'Ancien Régime l'ignorèrent ${ }^{1}$, c'est que Cinq-Mars-la-Pile fut aussi jusqu'au début du $\mathrm{XX}^{\mathrm{e}}$ siècle l'un des principaux centres de production de meules de moulin en France. De prime abord, le sujet pourrait paraître accessoire. Il n'en est rien. La fabrication des meules a constitué jusqu'à la veille de la Première Guerre mondiale un important secteur industriel, dont dépendaient la qualité et la quantité des farines produites et donc du pain : sans bonnes meules de moulin, la France n'aurait jamais été le pays du " meilleur pain du monde " (Steven Kaplan). Une meule taillée dans une mauvaise pierre avait non seulement une piètre productivité, mais en plus elle semait, en tournant, du sable et du gravier qui infestaient la farine, et qui vous limaient les dents aussi sûrement qu'une râpe d'acier. Pour éviter ces inconvénients, rien ne valait de bonnes pierres en "meulière ", un silex semblable à une éponge, dont la France possédait les meilleurs et les plus grands gisements du monde : en Bassin Parisien, autour de La Ferté-sous-

1. BnF, Cinq Cents de Colbert, 277 : COLBERT DE CROISSY, Charles, Rapport à faire au Roy et à nos seigneurs de son Conseil royal de l'estat de la généralité de Tours, 1664. Voir encore Mémoire sur la généralité de Tours, dit de MiromesniL, 1698, d'après un renseignement aimablement fourni par Brigitte Maillard. Cette méconnaissance est classique en France où, contrairement à d'autres pays européens, les autorités ne prirent que tardivement conscience de l'importance de l'industrie meulière. Il faut attendre le milieu du XVIII ${ }^{\mathrm{e}}$ siècle et la naissance de la géologie en tant que science, pour voir paraître les premières publications sur le sujet. 
Jouarre et d'Épernon; en Poitou, dans la forêt de Moulière; en Dordogne autour de Domme et de Bergerac; et enfin en Touraine, à Cinq-Mars-la-Pile et dans les communes environnantes.

\section{Figure 1 - Vue générale de Cinq-Mars-la-Pile vers 1900}

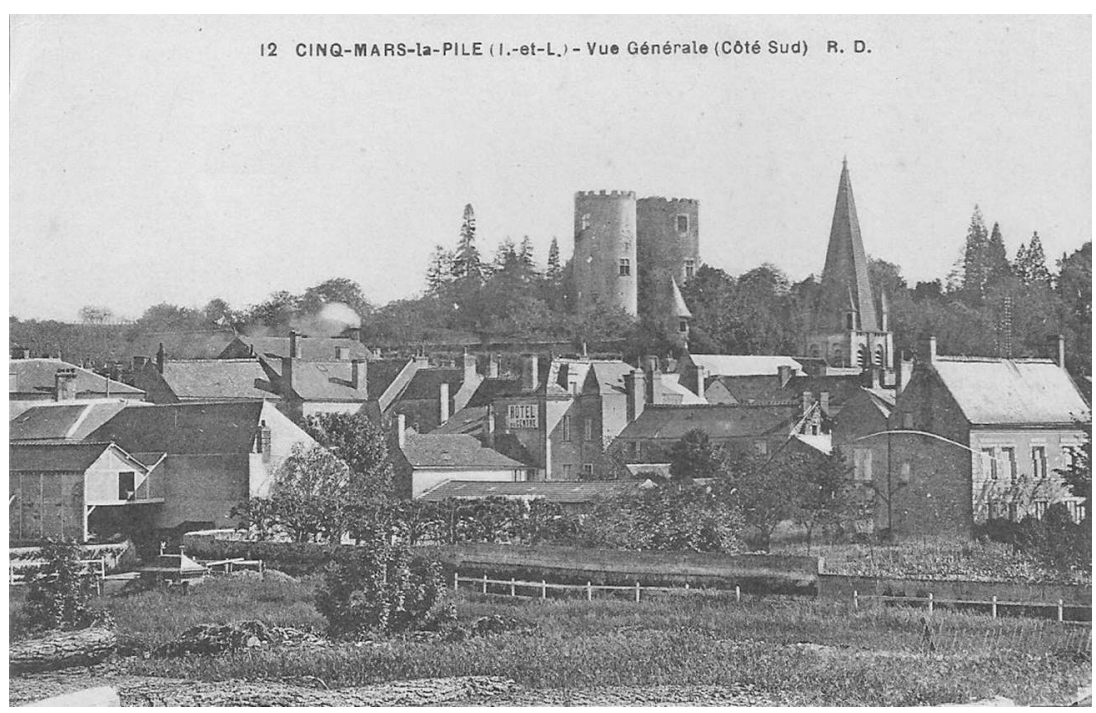

Les carrières de meules de Cinq-Mars-la-Pile ont déjà fait l'objet de plusieurs publications par des historiens locaux, Dorothée Kleinmann et Benoît Deffontaines ${ }^{2}$. Partant des archives d'entreprises en sa possession, Benoît Deffontaines a ainsi pu retrouver les lieux de production, reconstituer les procédés de fabrication, identifier le personnel et, enfin, dessiner l'aire de commercialisation de la principale firme cinq-marsienne, l'entreprise Brisgault frères, au cours des années 1890-1900. À cette époque, la société Brisgault exporte sa production à travers toute la France et principalement en Val de Loire et en Bretagne. Si l'histoire contemporaine des carrières de Cinq-Mars est donc bien explorée, les éléments sur les temps anciens faisaient par contre cruellement défaut. Le site surgissait de nulle part, en pleine maturité, ce qui supposait bien évidemment une plus grande

2. DEFFonTAINEs, Benoît, "La production et la commercialisation de meules de moulins à Cinq-Mars-la-Pile (Indre-et-Loire) ", Cahiers de l'Association des Amis des moulins de Touraine, mai 2002, n 1, 32 p., texte à nouveau publié dans BARBOFF, Mouette, SigAuT, François, GRIFFIn, Cozette et KREMER, Robert (dir.), Meules à grains. Actes du colloque international de La Ferté-sous-Jouarre, 16-19 mai 2002, Paris, Ibis Press-Maison des Sciences de l'Homme, 2003, p. 294-311. Voir aussi KLEINMANN, Dorothée, " La fabrication de meules à Cinq-Mars-la-Pile (Indre-et-Loire) ", Bulletin de la Société des Amis du Vieux Chinon, t. vIII, 1984, p. 1107-1116. 
ancienneté que celle qu'on lui accordait traditionnellement. Aussi, dans le cadre d'une enquête touchant toutes les grandes carrières de meules européennes, avons-nous lancé quelques coups de sonde à travers les registres des notaires de Cinq-Mars, conservés aux Archives départementales d'Indre-et-Loire. Ces registres se sont révélés une véritable mine : alors que souvent les carrières de meules ne livrent au mieux qu'un acte ici ou là, voire aucun, les 26 volumes dépouillés à Tours ont fourni 140 actes directement en rapport avec l'extraction ou la commercialisation des moullages, pour reprendre un terme de l'époque. Le plus ancien acte remonte à 1605 et le plus récent à 1672, conformément au programme de recherches que nous nous étions fixé, se focaliser sur les registres cinq-marsiens les plus anciens $^{3}$. Il en est sorti l'image d'un site précoce, aux structures complexes rivalisant durant le "Grand Siècle " avec les meulières les plus étendues du royaume, et qui participa directement à l'amélioration du pain quotidien des habitants de la Bretagne et des pays de l'Ouest.

\section{Contrôler les trésors du sous-sol : les permissions de fouilles}

Le 28 avril 1637, comparaissent devant maître Bureau, notaire à CinqMars, honorable Anne Morier, bourgeoise de la ville de Tours, et maître Pierre Marseille, " perrier de pierre de moullage " demeurant au village de Cinq-Mars. La dame Morier consent à ce que le maître artisan et sa femme fassent " tirer de la pierre de moullage au dedans d'un quartier de vigne a elle appartenant, situé près du lieu de la Houbellière et ou ledit Marseille a deja commancé a tirer de la pierre ". En échange, le tailleur de pierres payera au propriétaire de la parcelle 4 livres 10 sols pour chaque meule qu'il fabriquera, et ce pendant deux ans. Une fois l'exploitation terminée, maître Marseille s'engage à " recombler les fosses quils auront faites pour tirer lesdites pierres, et en outre replanteront de la plante de vigne dans ladite pièce de vigne de la Houbellière ${ }^{4}$ ". Ce type d'acte s'appelle une permission de fouilles. Elle autorise un meulier professionnel à éventrer le fond et le tréfond d'une parcelle autant qu'il le désire, même si elle appartient à autrui, moyennant le versement au propriétaire d'une indemnité proportionnelle à la production de la carrière. Qu'il soit bourgeois, ecclésiastique ou simple roturier, le détenteur de la parcelle ne peut en aucun cas s'opposer aux visées des meuliers. Depuis un édit promulgué en 1601 par Henri IV, et plusieurs fois renouvelé, le droit français reconnaît en effet le travail des meuliers comme une activité prioritaire, à laquelle nul propriétaire du sol ne peut s'opposer; seuls les membres de la noblesse

3. Ont ainsi été dépouillés aux archives départementales d'Indre-et-Loire, les registres 3E 42/46 à 57 (J. Boullemer, 1605-1634); 3E 42/15 (N. Buray, 1626-1644); 3E 42/68 (M. Coustellet, 1633-1641); 3E 42/20 à 27 (M. Bureau, 1635-1674); 3E 42/115-116 (U. Aymer, 1643-1660); 3E 42/16 (M. Porcher, 1646-1662).

4. Arch. dép. d'Indre-et-Loire, 3E 42/20. 
dérogent à la règle ${ }^{5}$. Ce système permet ainsi aux meuliers d'avoir un libre accès à la ressource minérale, tout en s'assurant que celle-ci ne sera pas gaspillée par des artisans improvisés, ou monopolisée par des membres de l'élite sociale, puisque seuls des meuliers professionnels et les marchands de meules peuvent se prévaloir des édits royaux. En échange de leur occupation des lieux, les meuliers versent aux propriétaires des parcelles muées en carrières une redevance de 2 livres par meule produite dans les années 1620, un chiffre qui passe à 4 livres dans les années 1640 puis à 6 et enfin 9 livres dans les années 1660. Cette redevance équivaut à $10 \%$ ou $20 \%$ du prix des meules et peut s'avérer une poule aux œufs d'or pour le propriétaire du sol : si les fosses ouvertes chez lui produisent plusieurs centaines de meules, elles lui rapporteront largement plus que la valeur de son terrain, et même une fortune si le chantier produit des milliers de meules.

La présence de pareilles permissions de fouilles témoigne de l'ampleur des carrières de Cinq-Mars et de leur ancienneté. Nous n'en avons en effet retrouvé de similaires que sur les plus grands gisements meuliers de France, à commencer par ceux de La Ferté-sous-Jouarre (Seineet-Marne), où besognaient 4000 ouvriers meuliers à la fin du XVIII ${ }^{\mathrm{e}}$ siècle $^{6}$. Sur le site tourangeau, ces permissions de fouilles apparaissent dès les plus anciens registres notariés conservés à Cinq-Mars, en 1605, lorsqu'un journalier autorise deux meuliers à mettre son pré sens dessus-dessous pendant deux ans, et même indéfiniment s'ils devaient trouver davantage de pierre exploitable à l'issue des deux ans ${ }^{7}$. En agissant ainsi, les artisans cinq-marsiens s'assurent l'exclusivité de l'accès aux pierres qu'ils convoitent. Du coup, ils multiplient les permissions, même sur les terrains vierges de tout sondage, afin de quadriller le territoire de la communauté. Entamé au moins depuis le début du " Grand Siècle ", le mouvement s'amplifie durant les années 1640 à 1660 incluses, qui voient à elles seules trente-cinq permissions accordées. Faut-il voir dans cette soudaine ruée une réaction à une menace extérieure? Les carrières de La Ferté-sous-Jouarre connaissent exactement le même phénomène au même moment : les meuliers profitent alors de l'endettement paysan résultant du " siècle de fer " pour contrôler étroitement l'accès au gisement ${ }^{8}$. Cette crue de permissions peut aussi résulter d'une conjoncture purement locale : en 1644, le seigneur de Cinq-Mars poursuit en justice deux perriers parce qu'ils ont ouvert dans ses parcelles « des grandes fosses et ils ont tiré quantité de pierre de moulage sans la permission de mondit seigneur ${ }^{9}$ "; il est possible que ce procès ait amené la pro-

5. JOURDAN, DECRUSY et ISAMBERT, Recueil général des anciennes lois françaises, Paris, Belin, 1825 , tome XV, p. 255.

6. BELMONT, Alain, La Pierre à pain. Les carrières de meules de moulins en France, du Moyen Âge à la révolution industrielle, Grenoble, Presses universitaires de Grenoble, 2006, tome 2.

7. Arch. dép. d'Indre-et-Loire, 3E 42/46, permission du 23 avril 1605.

8. Belmont, Alain, La Pierre à pain... op. cit., t. 2, p. 67.

9. Arch. dép. d'Indre-et-Loire, 3E 42/21, 13/12/1644. 
fession à une plus grande prudence. Au début du XvII ${ }^{\mathrm{e}}$ siècle, le recours aux permissions n'était en effet pas systématique, comme en atteste un acte du 11 novembre 1622 par lequel le bien nommé Jehan Le Perrier officialise le droit d'extraire des meules dans une parcelle " en laquelle y a plusieurs fousses dans lesquelles on a tiray autrefois des pierres de moullage ${ }^{10}$ ". Dans le lot des permissionnaires, certains meuliers comme les Marseille ou les Duvaux se taillent la part du lion, ce qui oblige les moins bien lotis qu'eux à lier des contrats d'association ou de souslocation, voire à acheter des droits d'accès au gisement : ainsi, le 15 juin 1645, Clément Duvaux vend à Pierre Viber " une quarte de terre située au lieu du Champ Simon apartenant a Urban Racault, dans laquelle pièce [il pourra] y tirer par ledit Viber de la pierre de moullage pendant le temps du bail qui en a été fait par ledit Duvau ", à charge de payer 4 livres par meule produite et du don d'une meule ${ }^{11}$.

\section{Tableau 1 - Les permissions de fouilles accordées aux meuliers de Cinq-Mars, de 1605 à 1672}

\begin{tabular}{|c|c|c|c|c|c|c|c|c|}
\hline $1605-$ & $1610-$ & $1620-$ & $1630-$ & $1640-$ & $1650-$ & $1660-$ & $1670-$ & TоTAL \\
1609 & 1619 & 1629 & 1639 & 1649 & 1659 & 1669 & 1672 & \\
\hline 3 & 1 & 7 & 4 & 10 & 14 & 11 & 0 & 50 \\
\hline
\end{tabular}

\section{Une nuée de meulières}

Permissions de fouilles, ventes de meules et associations entre meuliers dessinent la carte des carrières de Cinq-Mars. Elles s'avèrent particulièrement nombreuses puisque la documentation réunie en cite sur 36 toponymes : à La Houbellerie, aux Jarriats, aux Roberges, à Racault, au bois du Prieur, à La Gautellerie, etc. Certains sites entament le coteau dominant la rive droite de la Loire, où les bancs de pierre exploitables affleurent naturellement, ainsi que les versants de la vallée du Breuil, un ruisseau traversant toute la commune du nord au sud. Mais on trouve aussi des " ateliers " de meules sur le plateau constituant toute la partie septentrionale du territoire communal, comme au lieu de La Houbellerie, où se situent les plus grands chantiers de Cinq-Mars, fort de ses 12 permissions de fouilles accordées. Son challenger se trouve au sommet de la vallée du Breuil, au bois du Prieur, où les fosses percent le terrain sur plusieurs hectares. En somme, plus qu'une carrière isolée, Cinq-Mars présente un véritable bassin carrier, qui se prolonge sur les communes voisines de Mazières-de-

10. Arch. dép. d'Indre-et-Loire, 3E 42/52. L'expression " on a tiré autrefois ", utilisée en 1622, pourrait-être un indice de l'existence des meulières de Cinq-Mars dès la période médiévale. Leur degré d'organisation dès le règne de Henri IV milite également pour un passé déjà vieux de plusieurs siècles.

11. Arch. dép. d'Indre-et-Loire, 3E 42/21. 
Touraine $^{12}$, de Pernay ${ }^{13}$, et au-delà de la Loire et du Cher, jusqu'à Villandry et Savonnières ${ }^{14}$.

La visite des sites s'avère un peu décevante. Au lieu des fronts de taille monumentaux, percés de milliers d'alvéoles d'extraction circulaires, comme on peut en voir à Claix en Charente-Maritime, au Mont Vouan en Haute-Savoie, ou aux Écouges en Isère, pour ne citer que quelques exemples ayant fait l'objet de fouilles archéologiques récentes ${ }^{15}$, les carrières de CinqMars-la-Pile ont des allures de champ de bataille de la Première Guerre mondiale, et alignent une multitude de fosses ovoïdes semblables à des trous d'obus, séparés les uns des autres par des cordons de déchets de taille (les haldes), parfois maintenus par des murets de soutènement. Ainsi se présente le bois du Prieur, où plusieurs centaines de fosses relativement bien conservées s'étirent sur plusieurs hectares. La plupart se caractérisent par leur petite taille, mesurant 5 à 10 mètres de long pour 3 à 5 mètres de large et 1 à 2 mètres de profondeur. Au nord, la zone d'extraction est délimitée par un grand fossé de 2 mètres de large. À 1,5 kilomètre de distance et de l'autre côté de la vallée du Breuil, les carrières des Jarriais présentent un paysage analogue mais de moindre envergure que le bois du Prieur. On remarque seulement ici la présence de fosses plus importantes, allant jusqu'à une trentaine de mètres de longueur. Ailleurs, et notamment autour du hameau de la Houbellerie où se situait l'essentiel des sites en activité au XVII ${ }^{\mathrm{e}}$ siècle, les meulières ont fait les frais de la restauration des terrains et de leur reconversion agricole exigées par les permissions de fouilles; elles ont purement et simplement été gommées du paysage, qui ne montre plus que des étendues labourées, d'anciennes vignes et des vergers. Si l'on extrapole aux 36 toponymes évoqués par les sources, l'ampleur des chantiers conservés au Bois du Prieur, nous obtenons un bassin carrier d'ampleur industrielle, ayant forcément fourni plusieurs dizaines de milliers de meules. Voire davantage?

\section{Une pierre à " moulages "}

Le fond des fosses subsistantes ainsi que les haldes qui les bordent abondent en déchets de taille. La pierre sur laquelle les meuliers jetèrent

12. Arch. dép. d'Indre-et-Loire, 3E 42/22, permission de tirer des meules donnée par Pierre Caillard, de Mazières, 25 novembre 1652.

13. Arch. dép. d'Indre-et-Loire, 3E 42/68, procuration de vente du 8 janvier 1635.

14. Arch. dép. d'Indre-et-Loire, 3E 42/68, vente par Sébastien Poitier, de Savonnières, 30 juin 1639.

15. Belmont, Alain, Les carrières de meules de Claix, lieu-dit Les Meulières, département de la Charente. Rapport de fouilles programmées, SRA Rhône-Alpes, 2008, 4 volumes, 503 p. ; Belmont, Alain, Minvielle, Nicolas, Carrière de meules de La Molière à Vachat, Viuz-en-Sallaz (Haute-Savoie). Rapport de fouilles programmées (campagne 2010), SRA Poitou-Charentes, 2013, 355 p.; BELMONT, Alain, La carrière de meules des Écouges, lieu-dit Les Molières, commune de Saint-Gervais. Rapport de fouilles programmées, SRA RhôneAlpes, juin 2005, 79 p. 
Figure 2 - Un sous-bois aux allures de champ de bataille de Verdun : les fosses du Bois du Prieur

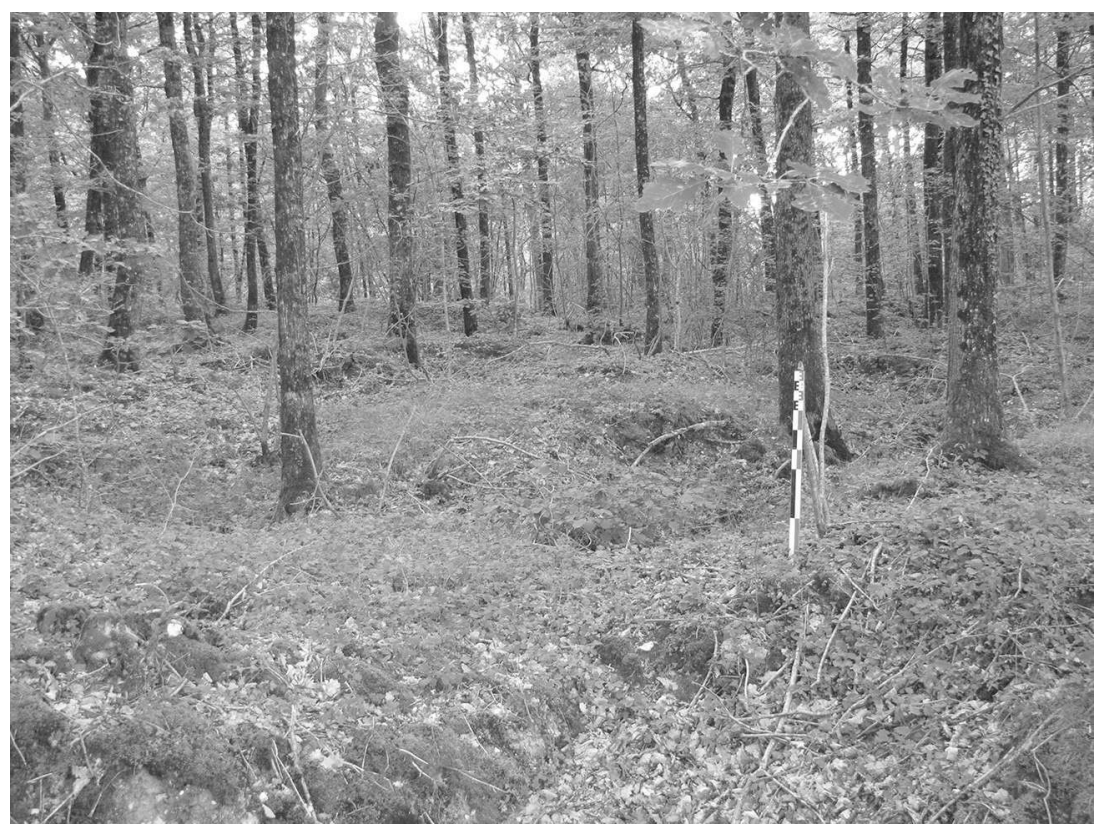

leur dévolu est décrite au XIX ${ }^{\mathrm{e}}$ siècle comme un "silex molaire ${ }^{16}$ " ou un " silex meulier ${ }^{17}$ ". Datant du Sénonien (fin de l'ère secondaire), ces silex forment des amas de blocs discontinus de plusieurs mètres d'épaisseur, empâtés dans une couche d'argile coiffant les dépôts calcaires si communs en Touraine. Extrêmement dur, et localement creusé d'alvéoles lui conférant une abrasivité naturelle, le silex cinq-marsien compte parmi les meilleures pierres aptes à la mouture des grains. Les meules taillées dans ce matériau auront une durée de vie d'au moins une dizaine d'années, contre à peine deux trois ans pour un mauvais grès, et surtout elles ne sèmeront pas du sable et du gravier dans la farine. Les grains de froment moulus entre des pierres de Cinq-Mars ressortiront propres et, une fois passés au tamis ou au blutoir pour que le son en soit éliminé, donneront une farine d'un blanc immaculé.

À en juger par les différences d'indemnités payées à une période donnée aux propriétaires des parcelles suivant leur localisation, - par exemple une livre de plus pour des pierres du Bois du Prieur par rapport à celles des Jarriais -, les meuliers d'autrefois et leurs clients distinguaient plu-

16. BARRUEL, G., Cours élémentaire de géologie, minéralogie et géognosie, Paris, André, 1843, p. 402.

17. CUVIER, Frédéric Georges, Dictionnaire des sciences naturelles, Paris, Le Normant, 1829, tome LIV, p. 201. 
Figure 3 - Le silex de Cinq-Mars-la-Pile

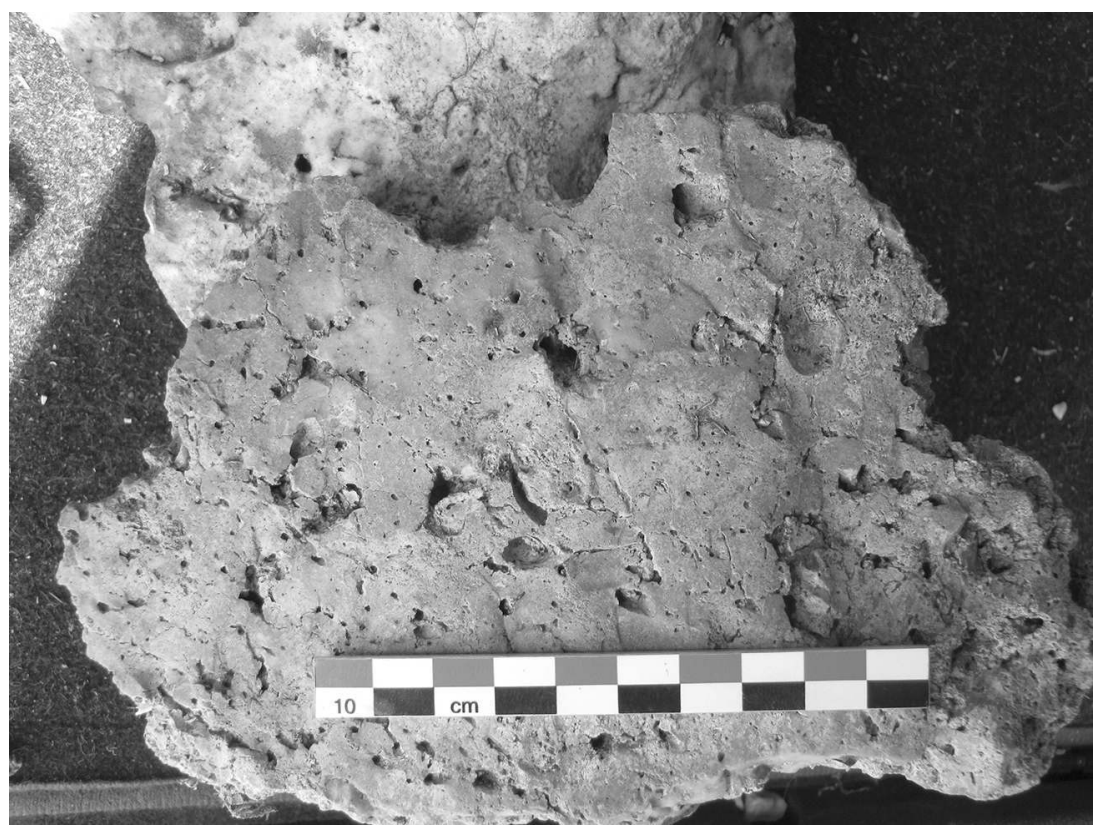

sieurs qualités de pierres au sein du gisement. Et de fait, le 26 octobre 1630 Guillaume Loizeau, meunier à Meung-sur-Loire (Loiret), prend bien soin de préciser à ses fournisseurs, Pierre et Jacques Duvaulx, que les deux meules qu'il leur achète devront être " a la coulleur dun quartier qui a este convenu dont ledit Loizeau a emporté un echantillon ${ }^{18}$ ". Ce même acte précise que les Duvaulx livreront au meunier de Meung non pas deux meules monolithes, mais " deux ronds de pierre bonnes a faire meulle sans boitier fournis de quartiers". Ces meules en quartiers - ou en carreaux, pour reprendre une expression du bassin parisien -, constituent l'unique production des carrières de Cinq-Mars. La technique consiste à assembler en " ronds", autour d'un bloc central percé (le " boitier "), des pavés de silex de diverses dimensions, la "moulange " ou le "moulage ", liés entre eux par du plâtre ${ }^{19}$. Ces quartiers ou carreaux de "moulage " sont taillés dans les fosses à même les blocs de silex, en employant des coins en fer et surtout des marteaux et des pics, qui s'usent à une vitesse vertigineuse compte tenu de la dureté de la pierre et qu'il faut régulièrement affûter ou remplacer : ainsi le 7 juin 1632, René Roux, marchand en armes blanches de Cinq-Mars, reçoit de Lucas Marguet, marchand perrier en pierres de mou-

18. Arch. dép. d'Indre-et-Loire, 3E 42/56.

19. Arch. dép. d'Indre-et-Loire, 3E 42/21, 5/8/1647, Pierre Dunant recevra de son client "le plastre qu'il conviendra pour faire lesdites meules". 
lage, 7 livres 12 sols " pour ses vacations d'un an et avoir fourni plusieurs pointes à ses marteaux ${ }^{20}$ ".

Les avantages de cette technique en " pièces détachées " sont nombreux. Elle permet de varier les qualités de pierre au sein d'une même meule, en alternant par exemple les parties dures et les parties plus

\section{Figure 4 - Divers assemblages de meules en carreaux}

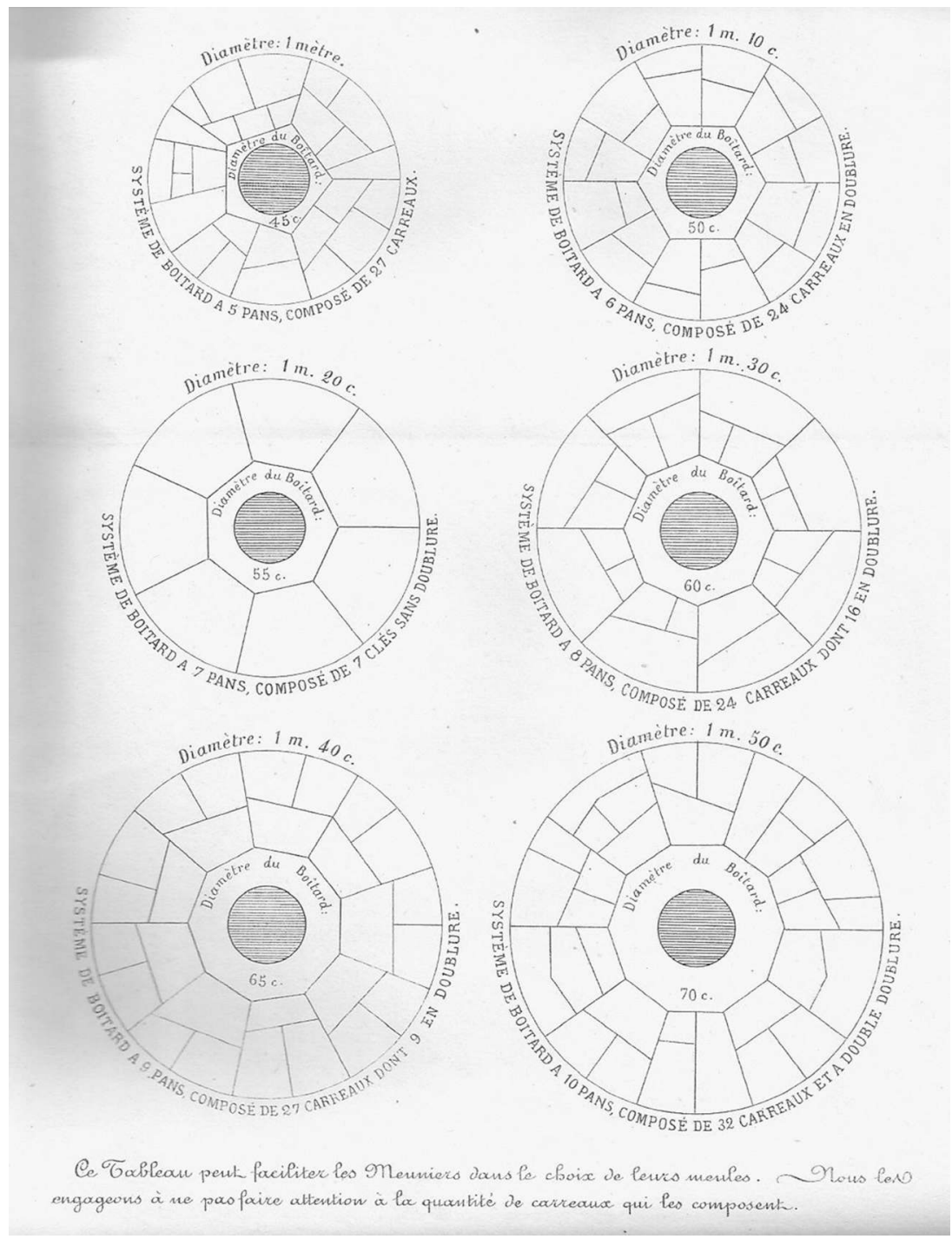

20. Arch. dép. d'Indre-et-Loire, 3E 42/57. 
tendres, pour mieux répondre aux efforts mécaniques exigés par la mouture du grain. Surtout, les meules en carreaux s'avèrent nettement plus faciles à transporter que des meules monolithes, surtout si elles sont assemblées sur les rives de la Loire ou, mieux encore, à proximité des moulins des clients $^{21}$. Compte tenu du fait que les "ronds de moulages " de Cinq-Mars mesurent 6 pieds de diamètre " selon la coutume du pais ${ }^{22}$ ", soit 1,95 mètre, et jusqu'à 7 pieds et demi pour les plus grosses ${ }^{23}$, soit 2,44 mètres de diamètre, leur poids atteint deux à trois tonnes. On imagine sans peine la difficulté à véhiculer pareilles masses, avec les moyens de transport du siècle de Louis XIII et de Louis XIV. Mieux valait charrier des carreaux de quelques dizaines de kilos que des mastodontes d'une seule pièce.

\section{Le peuple des carrières}

Trois catégories de personnes gravitent autour des carrières de meules : les " hommes de peine ", les " maîtres perriers ", et les " marchands perriers en pierre de moullage ". Les hommes de peine n'apparaissent qu'en filigrane des actes notariés. Ces journaliers remplissent pourtant un rôle essentiel. Il leur incombe de faire la " découverte", autrement dit d'excaver les sédiments recouvrant les blocs de silex pour les rendre accessibles aux meuliers. La couche de " morts-terrains " pouvant atteindre jusqu'à 7 mètres d'épaisseur à Cinq-Mars, les travaux de découverte représentent une entreprise considérable. De même, on confie à ces forçats de la pierre le soin d'évacuer des chantiers les déchets de taille, les "moellons", comme les appellent les textes, puis de les empiler du mieux possible afin qu'ils encombrent au minimum un espace de travail déjà exigu. Enfin, sur eux repose le rebouchage des fosses lorsque la permission de fouilles l'impose, ce qui est le cas dans la majeure partie des carrières. Certains d'entre eux parviennent à se hisser jusqu'au rang de meulier, comme ce Jean Racau, " homme de peine de Cinq Mars », qui s'associe le 19 mai 1629 avec le perrier Jacques Marguet, " pour tirer de la pierre de moullage des heritages leur appartenant au dedans de cette paroisse pendant cinq ans "; le journalier et l'artisan partageront par moitié les coûts et les recettes de leur association $^{24}$.

Le meulier par excellence, le maître dans l'art d'amoulanger, est donc le " perrier ", terme générique qui désigne en Touraine et en Anjou les

21. Arch. dép. d'Indre-et-Loire, 3E 42/15, accord infra judiciaire entre Pierre de la Tasche et Pierre et Clément Duvaux, 12 septembre 1644. "Lorsque les pierres ont été mises en rond, sur le port de la Maison Guesnon, il ne s'en est trouvé que huit et un quart et non dix." "

22. Arch. dép. d'Indre-et-Loire, 3E 42/46. Le 23 avril 1605, Mathurin Croix vend « un rond de pierre de moulage de six pieds ", avec son boîtier.

23. Arch. dép. d'Indre-et-Loire, 3E 42/47. Vente de 6 ronds de pierre de 7 pieds $1 / 2$ de diamètre, 28/?/1628 (le mois de cet acte n'est pas connu, le haut de la page ayant été grignoté par les rats).

24. Arch. dép. d'Indre-et-Loire, 3E 42/56. 
ouvriers de toutes sortes de carrières, notamment de calcaire et d'ardoise. Les registres dépouillés ont permis d'en recenser 53, dont 10 travaillant durant les années 1605-1620 et 27 oeuvrant au cours des années 16501660 , signe d'une progression de l'activité. Au contraire des grandes meulières d'ampleur régionale, dont chaque département français possède au moins deux ou trois exemplaires, les carrières de Cinq-Mars ne sont pas exploitées par un groupe restreint de familles contrôlant la totalité du gisement et du processus d'élaboration des meules, et encore moins par un seul clan comme c'est souvent le cas ${ }^{25}$. Ici interviennent une quarantaine de patronymes différents, les Viber, les Devisse, les Lucas (etc.) et surtout les Duvaux, les Marseille et les Marguet, qui forment des dynasties que l'on retrouve tout au long du XVII ${ }^{\mathrm{e}}$ siècle, et qui semblent avoir dominé d'une tête leurs confrères. À ces hommes reviennent la négociation des permissions de fouilles, la direction des travaux de déblaiement des couches à silex, l'extraction des blocs et enfin le façonnage et le montage des carreaux.

Considérés avec beaucoup d'égards par les notaires, qui n'hésitent pas à leur donner le titre de " maistres perrier de pierre de moulage " au début du siècle, ils voient peu à peu leur notoriété chuter, pour n'être plus traités que de " perriers à moullage ", voire simplement de "perriers " au fur et à mesure que l'on avance dans le temps. Chaque perrier exploite une ou plusieurs carrières à la fois, avec l'aide de ses fils et de quelques ouvriers. Lorsque les affaires marchent bien, il n'est pas rare de voir fleurir des associations entre plusieurs maîtres. Ainsi le 17 juin 1641, Pierre Duvaux le jeune et Jacques Marquis le jeune s'associent durant un an " pour tirer de la pierre de moullage des lieux ou ils prendront des perrières pour les faire fossoyer et les vendre et les délivrer aux marchands ". Pour en partager les débours, "ledit Duvau fournira les deux-tiers des ferrons [des outils] quil y conviendra et ledit Marquis en fournira un tier seulement attendu que ledit Duvau fournira un homme pour son domestique pour travailler avec lui "; les profits seront partagés à hauteur des investissements de chacun, deux tiers pour Duvaux et un tiers pour Marquis ${ }^{26}$. Simplicité des accords et brièveté de leur durée, telles sont les caractéristiques de ces associations entre perriers. Elles n'en traduisent pas moins un niveau d'élaboration des entreprises que l'on chercherait vainement dans la quasi-totalité des meulières françaises. Une fois de plus, seuls les grands sites parviennent à un tel degré de maturité.

Ces associations entre maîtres perriers constituent peut-être la réponse à une menace qui pèse sur leur métier à tous, la mainmise des

25. BELMONT, Alain, « La pierre et le pain. Les carrières de meules de moulins de Quaixen-Chartreuse (XVI ${ }^{\mathrm{e}-\mathrm{XVIII}}{ }^{\mathrm{e}}$ siècle) ", Histoire et sociétés rurales, $\mathrm{n}^{\circ}$ 16, 2001, p. 45-79. ID., "Une mine pour la farine. Les meulières de Berland (38) sous l'Ancien Régime ", La pierre et l'écrit, $\mathrm{n}^{\circ}$ 13, 2002, p. 37-68. ID., "Les carrières de meules de moulins ", dans GARDEL, MarieÉlise et BaIlly-MaITRE, Marie-Christine (dir.), La pierre, le métal. L'économie castrale en territoire audois, Carcassonne, Bulletin de la SESA, 2007, p. 39-50.

26. Arch. dép. d'Indre-et-Loire, 3E 42/20. 
marchands de meules. Le troisième et dernier acteur du théâtre meulier de Cinq-Mars-la-Pile s'incarne en une vingtaine de " marchands perriers en pierre de moullage ", qui apparaissent dès les premiers registres de notaires conservés aux Archives d'Indre-et-Loire, en 1605 (6 familles), et dont le nombre ne cesse de croître, pour atteindre 14 familles dans les années 1640-1650. Ces marchands, souvent anciens artisans meuliers comme les Duvaux et les Marguet, obtiennent quelques permissions de fouilles. Ils achètent ponctuellement quelques "ronds " de meules à tel ou tel perrier mais surtout, ils s'arrogent l'exclusivité de la production des artisans. Le mécanisme de leur mainmise est bien rodé et reproduit au détail près le même phénomène que celui mis en place au même moment sur le site géant de La Ferté-sous-Jouarre (Seine-et-Marne) : le marchand achète une ou plusieurs meules au perrier, qu'il lui règle en avance. Le perrier se retrouve alors endetté auprès du marchand, qui le force à signer une " association " ou un " pacte " par laquelle l'artisan s'engage, pour une ou plusieurs années, à ne travailler que pour son créancier. Par exemple, le $1^{\mathrm{er}}$ mars 1664, Jacques Marquet s'engage à vendre pendant deux ans "toutes les pierres de moullages bonnes et marchandes qu'il tirera et fera tirer dans la paroisse de Cinq-Mars " à Pierre Guerrier, " pour le prix de 18 livres prises sur lesdites perrieres payables a fin de livraison ${ }^{27}$ ". À cette même époque, les marchands vendaient 25 à 27 livres pièce les meules de moulins.

À force d'exclusivités, les perriers se retrouvent pratiquement évincés par les marchands de meules des circuits de commercialisation des pierres et voient filer sous leurs yeux près de la moitié des bénéfices qu'ils auraient pu retirer de leur travail. En fin de processus, le perrier devient un simple ouvrier du marchand, mal payé et corvéable à merci : en 1647, on apprend à la fin d'une vente de quatre meules que « Jacques Marquet, perrier de Cinq Mars, s'est obligé d'aller aider audit Vinant a faire et fassoner lesdits ronds moyennant dix sols par jour ${ }^{28}$ ". Déjà l'année précédente, Pierre Guerrier et Pierre Duvaux, marchands à Cinq Mars, avaient embauché Louis Marquis jusqu'à la Saint-Michel, " pour travailler de son estat de perreieur en les lieux et endroits ou lesdits le voudront faire travailler, en foi de quoi il recevra dix sols pour chaque jour quil travaillera a leur besogne ${ }^{29}$ ". Dix sous par jour : leur salaire était à peine supérieur à celui d'un journalier... Aussi les conflits ne manquent-ils pas entre les " marchands-patrons " et les ouvriers perriers : en janvier 1663, Pierre Grosset traîne en justice les meuliers Guillaume Sustras et Henri Delannoy parce qu'ils ont cessé de travailler dans la carrière où ils étaient censés oeuvrer sans discontinuer; ce à quoi Sustrans et Delannoy « ont fait réponse qu'ils ont toujours travailler a tirer des pierres, que s'ils ont cessé quelques jours sa esté pour travailler a leurs héritages [...] et que dès demain il yront travailler audit lieu du bois

27. Arch. dép. d'Indre-et-Loire, 3E 42/25.

28. Arch. dép. d'Indre-et-Loire, 3E 42/21, 10 mars 1647.

29. Arch. dép. d'Indre-et-Loire, 3E 42/21, 4 avril 1646. 
du Prieur "..., où ils ne s'étaient pas présentés depuis plus de deux mois, rétorqua le plaignant ${ }^{30}$.

Si durs soient-ils en affaires, les marchands de meules n'en sont pas moins des chevilles ouvrières essentielles aux pierres de Cinq-Mars, car ils leur ouvrent les horizons du grand commerce, ce qu'un simple meulier n'aurait guère été en pouvoir de faire.

\section{Une aire de commercialisation à l'échelle de l'Ouest de la France}

Les filets jetés dans la petite mer des registres des notaires cinq-marsiens ont ramené 37 actes de vente de meules et lettres de voiture, ce qui est beaucoup pour ce genre de sujet, mais qui ne constitue que la surface émergée d'un commerce forcément plus important; à preuve, cette « interpellation " dans laquelle le marchand Jacques Pourchier rappelle à l'ordre le meulier Michel Croillard " pour luy devoir et fournir un rond de pierre de moullage suivant marché verbal ${ }^{31}$ ". Le lot recueilli esquisse néanmoins à gros traits l'aire de commercialisation des meules de Cinq-Mars entre les règnes de Henri IV et de Louis XIV.

\section{Figure 5 - L'un des clients des perrières de Cinq-Mars : le moulin du Pot au Beurre, à Saint-Étienne-de-Chigny}

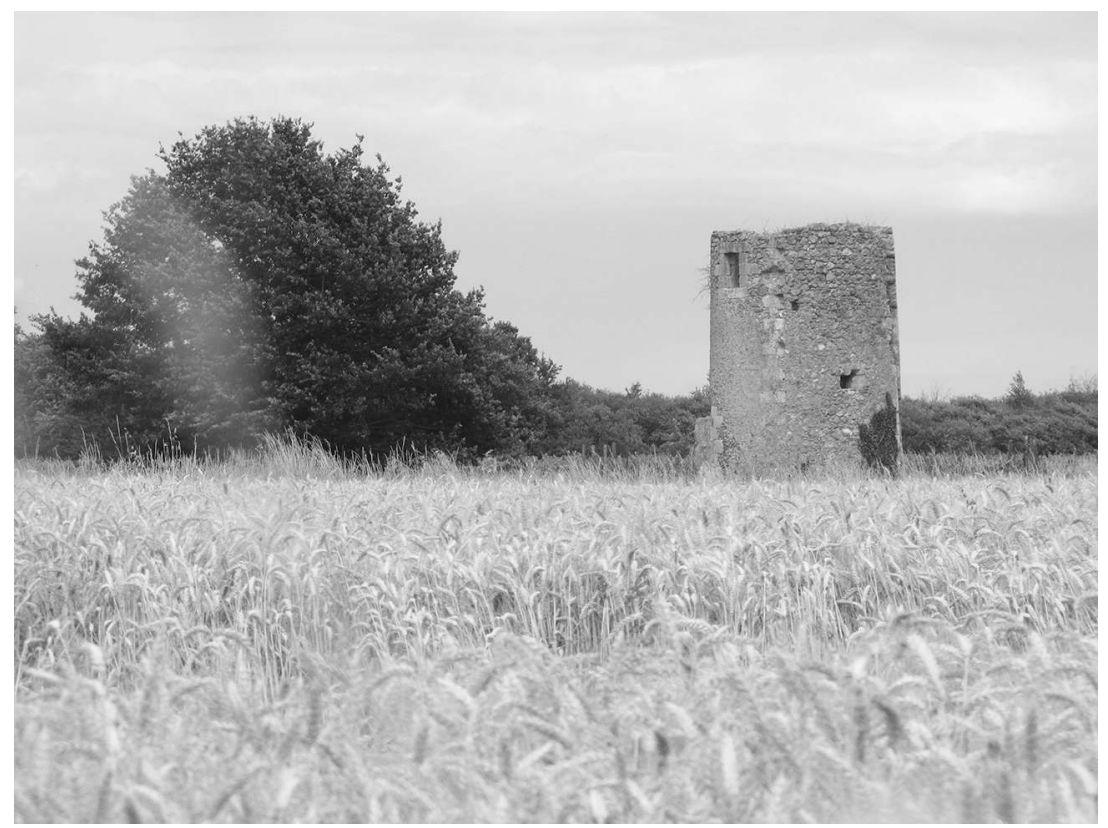

30. Arch. dép. d'Indre-et-Loire, 3E 42/24, 12 janvier 1663.

31. Arch. dép. d'Indre-et-Loire, 3E 42/20, acte du 6 mai 1636. 
Un premier cercle concerne les moulins installés à Cinq-Mars même et dans les communautés environnantes. Ainsi, le 10 février 1636, le marchand perrier Mathurin Croix vend au meunier de Cinq-Mars-la-Pile nommé Pierre Morlière, "trois ronds de pierre de moullage bonne et belle, de la taille de six pieds de roi ", que le meunier ira lui-même choisir au fond de la carrière avant leur livraison : "Lesquels seront veu et visité sur les perrières et dont ledit Croix fera la livraison audit Morlière d'ici deux mois ${ }^{32}$. " Lorsqu'ils n'habitent pas trop loin du gisement, les meuniers agissent toujours de même, préférant s'assurer sur place de la qualité des pierres qu'ils achètent, plutôt que d'hériter d'une meule mal montée, fendue ou que le meulier n'aurait pas fait " faire bien duement et mettre en estat de moudre de bled et faire farine ${ }^{33}$ ". Ces ventes directes aux meuniers demeurent relativement rares dans les registres des notaires, et concernent quasi exclusivement des localités de la région tourangelle : Tours bien sûr, Saint-Règle (Indre-et-Loire, près d'Amboise), Saché (près d'Azay-le-Rideau), voire des localités plus éloignées comme Chabris (Indre), à 40 kilomètres de CinqMars, La Chapelle-Blanche-Saint-Martin (38 kilomètres), Saint-Georges-surCher (56 kilomètres) et Romorantin-Lanthenay (116 kilomètres), le record en la matière étant tenu par Guillaume Loizeau, meunier au grand moulin de Meung-sur-Loire, près d'Orléans, qui n'a pas hésité à franchir 120 kilomètres pour être " de présent à Cinq-Mars ${ }^{34}$ ». En principe, au-delà de 50 kilomètres, le marchand de meules prend le relais des meuniers et démarche lui-même les clients potentiels : le 6 mars 1636, le marchand Jacques Pourcher s'entend ainsi avec le perrier Michel Croillard pour lui payer les meules qu'il lui doit, "sitot quil sera de retour de la ville de Selle en Berry ou il va en voyage $^{35} "$.

Lorsque la distance à couvrir reste raisonnable, le moyen de transport le plus utilisé demeure un char spécialement conçu pour porter des meules (un fardier), attelé à des bœufs : en 1652, Pierre Caillard autorise ses voisins meuliers à " passer et faire passer avec bœufs et charettes les pierres de moullages qui se tirent au lieu de Bourjachières ${ }^{36}$ "; de même, en 1655, le marchand Pierre Guerrier "sera tenu de les prendre [les meules] des lieux ou elles seront tirées jusqu'au lieu ou il pourra mener sa charette sans faire domage ${ }^{37}$ ". Par contre, sitôt que le voyage s'allonge, le chemin de terre laisse place à la voie d'eau. Cinq-Mars-la-Pile a la chance de se trouver non seulement juste en face de l'embouchure du Cher mais aussi

32. Arch. dép. d'Indre-et-Loire, 3E 42/20.

33. Arch. dép. d'Indre-et-Loire, 3E 42/21, 10 mars 1647.

34. Arch. dép. d'Indre-et-Loire, 3E 42/20, 28 décembre 1641 (Saint-Georges); 3E42/46, 23 avril 1605 (Saint-Règle); 3E 42/56, 17 décembre 1630 (Romorantin); 3E 42/52, 27 juin 1619 (Tours et La Chapelle); 3E 42/54, 26 décembre 1626 (Chabris); 3E 42/56, 13 septembre 1629 (Saché); 3E 42/53, 25 mars 1623 (Azay-le-Rideau); 3E 42/56, 17 décembre 1630 (Meung).

35. Arch. dép. d'Indre-et-Loire, 3E 42/20, acte du 6 mai 1636.

36. Arch. dép. d'Indre-et-Loire, 3E 42/22, acte du 25 novembre 1652.

37. Arch. dép. d'Indre-et-Loire, 3E 42/23, acte du 15 mars 1655. 
Figure 6 - L'aire de diffusion des meules en région Centre au XVII siècle

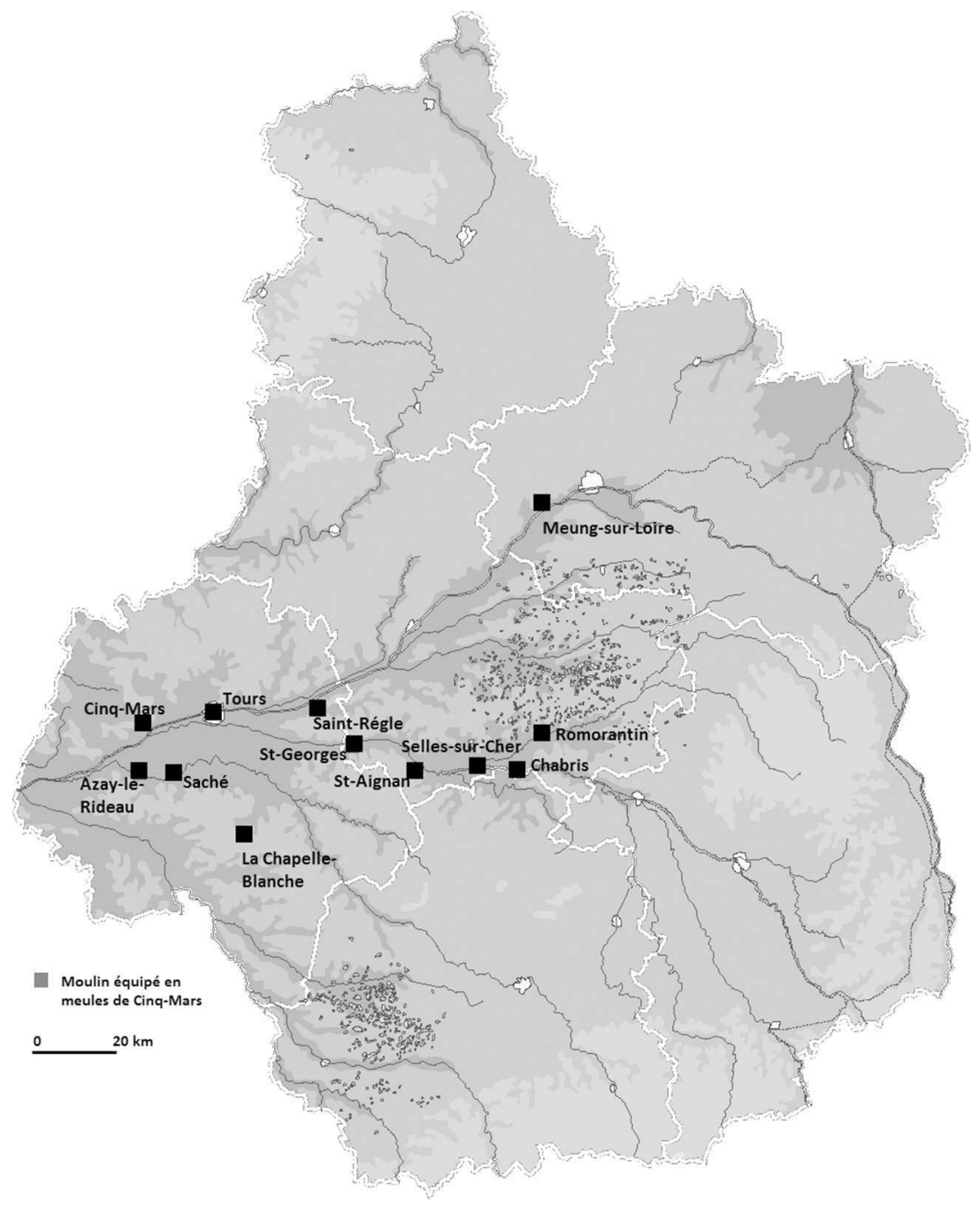

sur les berges même de la Loire, en rive droite du fleuve, et de disposer de tout un ensemble de " ports" - entendez des plages de graviers plus ou moins aménagées pour accueillir des chalands, le plus important d'entre eux semblant être le justement nommé port du Gravier, situé en contrebas de la pile gallo-romaine. Là viennent charger les bateaux des " voituriers par eau ", auxquels les marchands de meules confient leurs marchandises. Bon nombre de ces bateliers viennent de Saint-Étienne-de-Chigny, un village situé entre Cinq-Mars et Luynes. Mais deux ports se taillent la part 
du lion parmi les lettres de voiture. Le premier est Selles-sur-Cher, situé à 80 kilomètres de Cinq-Mars, qui paraît avoir servi de dépôt aux marchands cinq-marsiens tant il revient souvent dans la documentation ${ }^{38}$; de ce lieu, les flots de meules pouvaient irriguer les moulins du Berry, si l'on en juge au fait qu'un voiturier par eau reçut l'ordre en 1644 de porter les 10 " ronds " qui lui avaient été confiés " au port de Celles en Berry et autres ports audessoub $^{39}$ ". Le deuxième port en tête du lot, et de très loin, est Nantes. Sur les 37 ventes de meules et lettres de voiture trouvées aux Archives d'Indreet-Loire, 13 concernent l'ancienne capitale de la Bretagne et portent sur au moins 232 meules (55 meules pour la plus grosse livraison), le contenu de plusieurs chalands n'ayant malheureusement pas été précisé ${ }^{40}$. Ces lettres de voiture nantaises peuvent être classées en deux catégories. Soit elles confient au voiturier la vente des meules, souvent à l'exclusion de toute autre marchandise ${ }^{41}$; soit elles constituent des délégations de vente à des marchands nantais, marchands que l'on voit revenir à plusieurs reprises dans les textes, au point d'apparaître comme potentiels facteurs des entreprises cinq-marsiennes, même si le terme de facteur (un représentant de commerce, en charge d'un dépôt) n'est jamais explicitement employé par les actes : ainsi en 1671 et 1672, des marchands de Cinq-Mars font livrer au Nantais Étienne Béranger au moins trois chalands remplis de pierres de meules, à charge pour lui de les "faire mettre en rond audit Nantes " à ses frais ${ }^{42}$.

Depuis les dépôts de Selles-sur-Cher et de Nantes, les meules de CinqMars sont diffusées à travers les pays de Loire et la Bretagne. À preuve, le 21 février 1688, un commis et un meunier de l'abbaye Saint-Melaine de Rennes s'obligent " de faire et construire une meule ausdits moullins de Trublé, qui est celle de dessoubs du moullin a seigle de grandeur competante et de dix sept poulces d'épaisseur faicte et contruite de bon moullage de Sainct Marc pierre noire et du Jaulnay bien et duement joincte, assemblée et liaisonnée avec plastre, lequel moullage et plastre [le commis] s'oblige d'aller incessamment querir a Nantes et le faire conduire en cette ville $^{43}$ ». D'autres localités sont bien évidemment concernées, peut-être par centaines, mais la documentation que nous avons réunie ne permet pas de toutes les connaître. Par contre, nous disposons pour une période plus

38. Arch. dép. d'Indre-et-Loire, 3E 42/20 (5 novembre 1641) ; 3E 42/27 (20 avril 1672) ; 3E 42/24 (6 mars 1661).

39. Arch. dép. d'Indre-et-Loire, 3E 42/15, lettre de voiture du 12 septembre 1644.

40. Arch. dép. d'Indre-et-Loire, 3E 42/26 (21 octobre 11671); 3E 42/27 (24 mai 1672 et 23 mars 1672); 3E 42/25 (4 octobre 1665); 3E 42/24 (4 août 1663) ; 3E 42/52 (13 octobre 1620) ; 3E 42/57 (6 novembre 1632); 3E 42/21 (8 juillet 1647) ; 3E 42/68 (8 janvier 1635); 3E 42/68 (23 septembre 1637 et 30 juin 1639 et 16 juillet 1633); 3E 42/68 (8 janvier 1635).

41. Par exemple, Arch. dép. d'Indre-et-Loire, 3E 42/21, vente de 4 ronds et demi à Denis Buisson, payables à livraison lorsqu'il reviendra de Nantes (8 juillet 1647).

42. Arch. dép. d'Indre-et-Loire, 3E 42/26 (21 octobre 1671), 3E 42/27 (24 mai 1672) et 3E 42/27 (23 mars 1672).

43. Arch. dép. d'Ille-et-Vilaine, 3E 431, cité par Duran-VAugaron, L., " Technologie et terminologie du moulin à eau en Bretagne ", Annales de Bretagne, 1969, tome 76, n 2, p. 343-344. 
récente, d'une source sans équivalent pour dessiner les aires de commercialisation des meulières françaises : la grande enquête sur les moulins de France menée durant le règne de Napoléon I ${ }^{\text {er }} 44$. Réalisée en 1809 , cette enquête précise pour toutes les communes du pays la provenance des meules utilisées dans leurs moulins. Or, à la lumière de cette enquête, il apparaît que les meules de Cinq-Mars équipent une aire d'environ 400 kilomètres de long couvrant six départements du Centre, de Poitou-Charentes, des Pays-de-la-Loire et de la Bretagne : le Loir-et-Cher, les Deux-Sèvres, la Sarthe, le Finistère, l'Ille-et-Vilaine, et bien évidemment l'Indre-et-Loire. Dans ce dernier département, elles règnent en maître absolu; par contre, dès que l'on s'éloigne d'une centaine de kilomètres de Cinq-Mars, la pierre tourangelle se heurte à la concurrence féroce des meulières de la forêt de Moulière (Vienne), des Molières (Essonne) et surtout de La Ferté-sousJouarre (Seine-et-Marne), un monstre industriel qui, déjà au XVII ${ }^{\mathrm{e}}$ siècle, exporte sa production jusqu'en Amérique du nord.

Face à ses concurrents poitevins ou parisiens, tous de qualité égale ou supérieure, les "ronds de moullage " des bords de Loire ont pour avantage des prix deux à trois fois moins élevés. L'achat d'une meule aux perriers cinq-marsiens varie de 9 à 20 livres dans les années 1640-1660. Les marchands de meules eux, après avoir pris leur bénéfice, les cèdent à la même époque entre 22 et 33 livres pièce. S'ajoutent les frais de transport, qui montent entre 9 et 11 livres pour un " rond " livré à Selles-sur-Cher, et entre

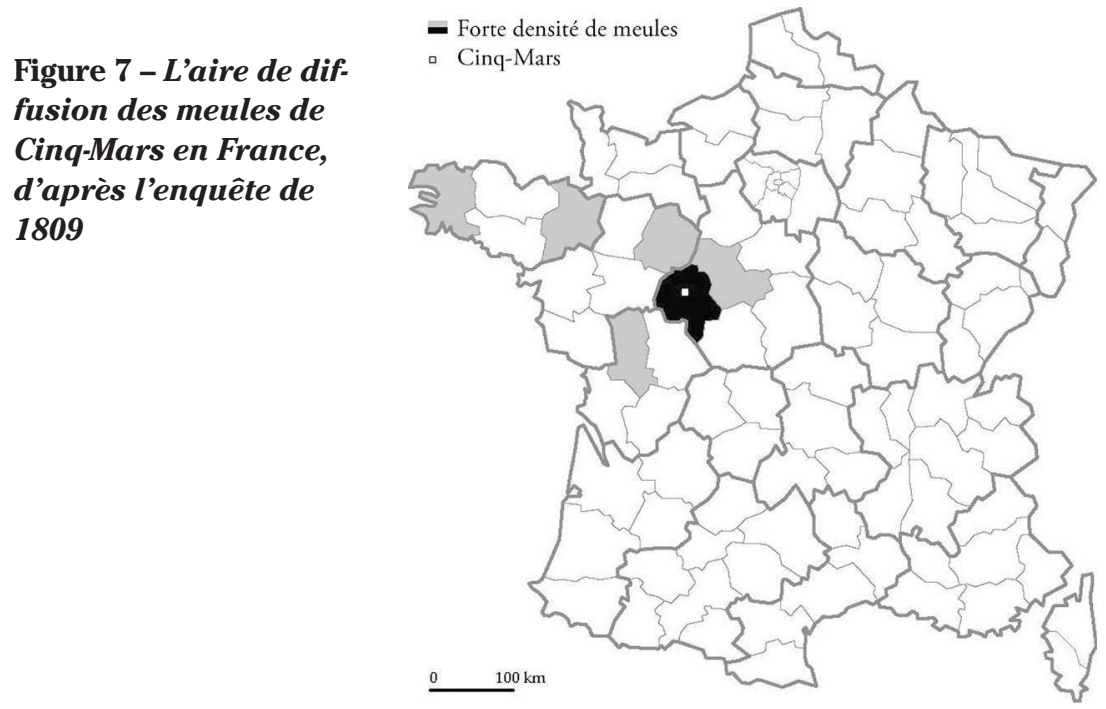

44. Arch. nat., F20/295 et 296. 
10 et 15 livres pour une meule voiturée jusqu'à Nantes. Tous frais compris, les meules livrées à Nantes reviennent à 40 livres pièce en $1635^{45}$. On reste très loin des 100 à 300 livres que peut coûter à la même époque une fertoise vendue dans l'estuaire de la Loire.

De bonne qualité, abordables pour tous les meuniers ou peu s'en faut, les pierres de Cinq-Mars-la-Pile portent de rudes coups aux autres meulières implantées en Pays-de-la-Loire et en Bretagne. Ainsi les meules du Guilvinec, extraites en Cornouailles, à l'extrémité ouest du département du Finistère, ne peuvent se mesurer à leurs semblables ligériennes. Le site du Guilvinec a pourtant dû connaître son heure de gloire : ouvert au sommet de l'estran, entre la pointe de Men Meur et l'entrée du port de pêche, il présente sur près d'un kilomètre plus d'une centaine d'alvéoles d'extraction et d'ébauches de meules monolithes ${ }^{46}$. Un peu plus loin, dans la lande et près de la chapelle de Saint-Trémeur, voici encore une vingtaine d'alvéoles d'extraction disposées en nids d'abeille, toutes à touche-touche ${ }^{47}$. Deux beaux sites en somme, sur lesquels les techniques employées par les anciens meuliers se lisent à livre ouvert. La pierre exploitée ici cumule plusieurs vertus : elle se compose d'un granite blanc, semé de cristaux de quartz relativement homogènes, et possède une forte densité alliée à une abrasivité idéale pour

\section{Figure 8 - La meulière de Men Meur, au Guilvinec (Finistère)}

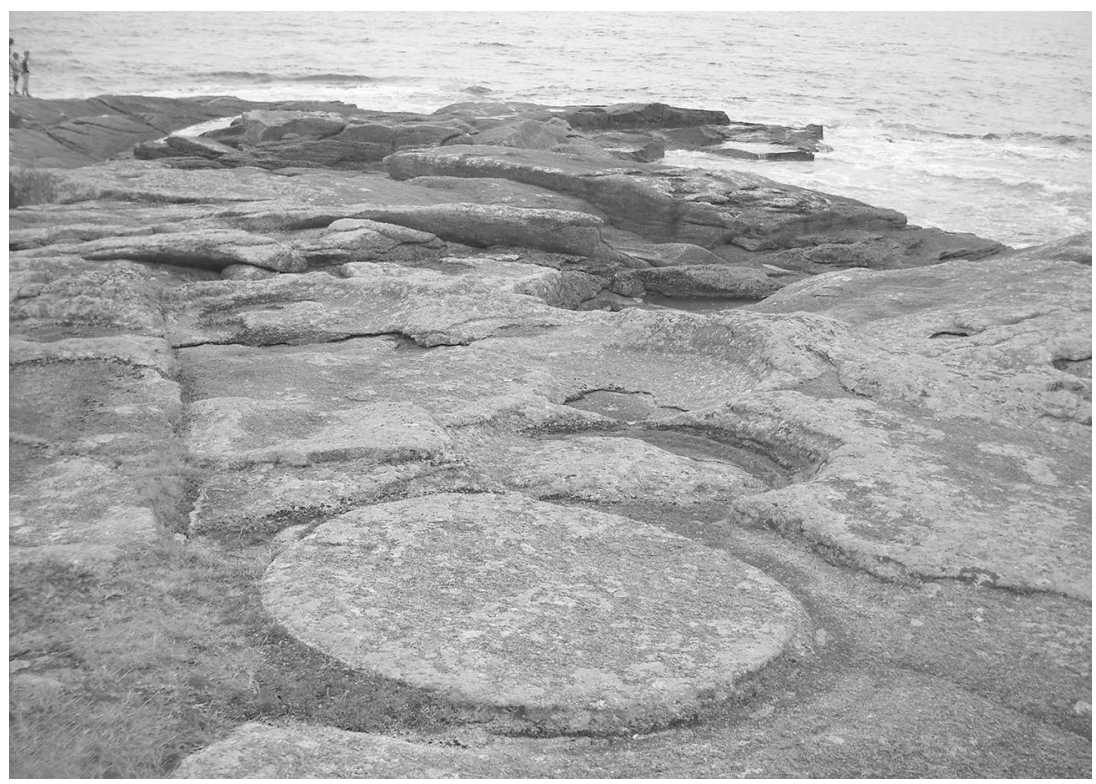

45. Arch. dép. d'Indre-et-Loire, 3E 42/68, acte du 8 janvier 1635.

46. N $47^{\circ} 47^{\prime} 31.8$; W 4ำ' $17^{\prime} 32.7$; $4 \mathrm{~m}$.

47. $\mathrm{N} 47^{\circ} 48^{\prime} 22.5 ; \mathrm{W} 4^{\circ} 17^{\prime} 40.5 ; 10 \mathrm{~m}$ 
moudre le grain. Elle montre par contre une granulométrie qui fait qu'en tournant à un ou deux tours/seconde à l'intérieur du moulin, cette pierre du Guilvinec devait finir par semer dans la farine des débris minéraux, que l'on retrouvait dans le pain. Aussi, sa clientèle a-t-elle dû se tarir peu à peu, tandis que les meules en carreaux de silex gagnaient ces contrées du Finistère. Et de fait, le diamètre des meules du Guilvinec mesurent 120 à 130 centimètres, soit des mensurations typiques du Moyen Âge et du début de l'époque moderne, alors que les meules de Cinq-Mars atteignent, on l'a vu, 1,95 mètre, une dimension classique de l'époque moderne. Le silex meulier de Cinq-Mars, d'Île-de-France ou des environs de Poitiers auront donc eu raison du vieux granite breton.

Derrière l'histoire des carrières de Cinq-Mars se cache un progrès fondamental : celui du meilleur pain qui se puisse produire. En s'échinant à tirer des blocs de silex des profondeurs du plateau tourangeau, puis en les montant en de savants assemblages circulaires, les meuliers cinq-marsiens ont permis à leurs contemporains de consommer un pain dépourvu de débris minéraux, et d'avoir ainsi plaisir à manger leur pain quotidien, alors qu'un grain moulu entre des mauvaises pierres aurait donné autant d'agrément que de croquer une poignée de sable. Ces pierres tourangelles procurèrent aussi aux consommateurs de l'Ancien Régime un pain plus abondant, puisque leur dureté autorisa au XvIII ${ }^{\mathrm{e}}$ siècle l'introduction de la " mouture économique ", un passage répété du grain entre les meules, qui permit de produire environ $15 \%$ de farine de plus qu'avec une mouture traditionnelle "à la grosse ", mais qui ne pouvait se faire qu'entre des meules très dures ${ }^{48}$. Elles furent en somme, à l'origine d'un progrès alimentaire.

Reste à savoir depuis quand cette industrie s'est mise en place ici. La mention au début du XVII ${ }^{\mathrm{e}}$ siècle de fosses abandonnées depuis longtemps d'une part, le haut degré d'organisation de l'exploitation perceptible dès les plus anciens registres des notaires d'autre part, militent en faveur d'une origine probablement médiévale. Un lien existerait-il entre la présence à cette époque de la cour de France en Val de Loire, et la mise en œuvre de carrières aptes à produire un pain raffiné, conforme à ses habitudes alimentaires? La recherche n'est pas épuisée, loin de là. Bien du grain reste à moudre pour les historiens et les archéologues qui seraient désireux de creuser le sujet plus avant.

48. KaPLAn, Steven, Les ventres de Paris, Paris, Fayard, 1988, 704 p. 


\section{RÉSUMÉ}

Ignorée de la plupart des historiens et des archéologues, la production des meules de moulins représenta jusqu'à la veille de la Première guerre mondiale un secteur industriel fondamental, car de sa bonne marche dépendait la quantité et la qualité du pain consommé par nos prédécesseurs. Parmi les grands gisements à la tête de cette industrie, se trouvait celui de Cinq-Marsla-Pile, en Indre-et-Loire. Connu par ses archives d'entreprises des $\mathrm{XIX}^{\mathrm{e}}$ et $\mathrm{xx}^{\mathrm{e}}$ siècles, son passé plus ancien demeurait pratiquement inexploré. Partant des plus anciens registres notariés conservés, l'article décrit le mode d'exploitation des carrières, le personnel mis en oeuvre, les productions et enfin l'aire de commercialisation d'entreprises qui irriguèrent de larges pans de l'Ouest de la France au XVII ${ }^{\mathrm{e}}$ siècle.

\section{ABSTRACT}

Ignored by most historians and archaeologists, the production of millstones was until World War One a very important industry, as the quality and quantity of bread depended on their maintenance and on further investment. One of the most important sites for millstone production in France was located at Cinq-Mars-la-Pile, in the Loire valley, near Tours. Whilst the nineteenth- and twentieth-century history of Cinq-Mars is well known, the earlier history of the site remained in large part unknown. Using a collection of 140 contracts dating from the seventeenth century, this paper describes the quarries, the stone cutters, the production and the trade area of businesses that distributed their wares in western France. 